\title{
ON UNIFORMIZATION OF SETS IN TOPOLOGICAL SPACES
}

\author{
BY \\ MAURICE SION
}

1. Introduction. Let $Q \subset X \times Y$ and $P$ be the projection of $Q$ onto $X$. A uniformization of $Q$ is a function $f$ on $P$ to $Y$ such that $f \subset Q$, i.e., for every $x \in P,(x, f(x)) \in Q$. Clearly with the aid of the axiom of choice, such a function can always be found. The problem, in general, is to find an $f$ that satisfies, in addition, certain conditions. Many authors have considered different aspects of this problem and we mention a few in the bibliography (see also $[5$, p. 55 and on $]$ ).

In case $X$ and $Y$ are Euclidean spaces one important result, due to Luzin [3] and Sierpinski [6] is the following: if $Q$ is Borel, then $f$ can be taken to be the complement of an analytic set in $X \times Y$. Kondô [2] has extended this result to the case where $Q$ is the complement of an analytic set, thereby deriving important properties of projective sets of class 2 .

In this paper, we take $X$ and $Y$ to be topological spaces satisfying certain conditions. If $Q$ is compact, then we obtain a uniformization $f$ that is a Borel function (Theorem 4.2). If $Q$ is analytic, then $f$ is a measurable function for a large class of measures on $X$ (Theorem 4.5).

2. Notation and basic definitions.

2.1. A non-negative integer $n$ contains all smaller non-negative integers, i.e., $m \in n$ iff $m<n$ and $m$ is a non-negative integer. Thus, 0 denotes both the empty set and the smallest non-negative integer.

2.2. $\omega$ denotes the set of all non-negative integers.

2.3. $f$ is a function on $A$ to $B$ iff $f \subset A \times B$ and, for every $x \in A$, there exists a unique $y(=f(x))$ such that $(x, y) \in f$.

2.4. $A$ is Borel $H$ iff $A$ belongs to the smallest family containing $H$ and closed under countable unions and differences of two sets.

2.5. $K(X)$ denotes the family of all compact sets in $X$.

2.6. $A$ is analytic in $X$ iff, for some Hausdorff space $X^{\prime}, A$ is the continuous image of a $K_{\sigma \delta}\left(X^{\prime}\right)$.

2.7. $\mathfrak{A}(X)$ denotes the family of all analytic sets in $X$.

2.8. If $A \subset X \times Y$, then $\pi A$ is the projection of $A$ onto $X$.

2.9. $Y$ satisfies condition (I) iff $Y$ is Hausdorff, regular, has a base whose power is at most that of the first noncountable cardinal, and every family of open sets in $Y$ has a countable subfamily with the same cover.

\section{Preliminary lemmas.}

3.1. If, for every $n \in \omega, A_{n}$ is compact, $A_{n+1} \subset A_{n}, \cap_{n \in \omega} A_{n} \subset B, f$ is a continuous function on $B$ to a Hausdorff space, then

Presented to the Society, January 29, 1960; received by the editors October 26, 1959. 


$$
f\left(\bigcap_{n \in \omega} A_{n}\right)=\bigcap_{n \in \omega} f\left(B \cap A_{n}\right) .
$$

Proof. Suppose $y \in \bigcap_{n \in \omega} f\left(B \cap A_{n}\right)$. Then, for every $n \in \omega, f^{-1}\{y\} \cap B \cap A_{n}$ $\neq 0$. Since $f$ is continuous on $B$, we have $f^{-1}\{y\}=B \cap C$ for some closed set $C$. Thus, for every $n \in \omega, C \cap A_{n} \neq 0$ and hence

$$
f^{-1}\{y\} \cap \bigcap_{n \in \omega} A_{n}=\bigcap_{n \in \omega}\left(C \cap A_{n}\right) \neq 0
$$

i.e., $y \in f\left(\bigcap_{n \in \omega} A_{n}\right)$.

The converse is obvious.

3.2. Suppose, for every $i \in I, A_{i} \subset X \times Y$ and, for every $x \in X$, the family $\bigcup_{i \in I}\left\{\pi^{-1}\{x\} \cap A_{i}\right\}$ forms a nest of compact sets in the product topology. Then

$$
\bigcap_{i \in I} \pi A_{i}=\pi \bigcap_{i \in I} A_{i}
$$

Proof. Let $x \in \bigcap_{i \in I} \pi A_{i}$. Then, for every $i \in I, \pi^{-1}\{x\} \cap A_{i} \neq 0$ and since the intersection of a nest of nonvoid compact sets is nonvoid, we have

$$
\bigcap_{i \in I}\left(\pi^{-1}\{x\} \cap A_{i}\right) \neq 0,
$$

i.e.,

$$
x \in \pi \bigcap_{i \in I} A_{i}
$$

The converse is obvious.

4. Uniformization of sets.

4.1. Theorem. Suppose $X$ is any space; $H$ is any family of subsets of $X$; $Y$ satisfies condition (I); $Q \subset X \times Y$; for every $x \in X, \pi^{-1}\{x\} \cap Q$ is compact; for every closed set $B$ in $Y, \pi((X \times B) \cap Q) \in H$. Then there exists a function $f$ on $\pi Q$ such that $f \subset Q$ and, for any open set $V$ in $Y, f^{-1}(V)$ is Borel $H$.

Proof. Let $\Omega$ be the first noncountable ordinal and $U_{i \in \Omega}\left\{U_{i}\right\}$ be a base for $Y$. Let, for $\alpha \subset X \times Y$ and $\beta \subset X \times Y$,

$$
P(\alpha, \beta)=(\alpha \cap \beta) \cup[((X-\pi(\alpha \cap \beta)) \times Y) \cap \beta] .
$$

Next, by recursion for every $i \in \Omega$, we define $A_{i}$ as follows:

$$
\begin{aligned}
A_{0} & =Q, \\
A_{i+1} & =P\left(X \times \bar{U}_{i}, A_{i}\right), \\
A_{i} & =\bigcap_{j<i} A_{j} \quad \text { if } i \text { is a limit ordinal, }
\end{aligned}
$$

where $\bar{U}_{i}$ denotes the closure of $U_{i}$. Finally, we set 


$$
f=\bigcap_{i \in \Omega} A_{i}
$$

and check the following points.

(i) $P(\alpha, \beta) \subset \beta$ and $\pi P(\alpha, \beta)=\pi \beta$.

(ii) If $i \in \Omega, j \in \Omega, j<i$, then $A_{i} \subset A_{j}$.

(iii) If $i \in \Omega$ and $x \in X$, then $\pi^{-1}\{x\} \cap A_{i}$ is compact.

(iv) If $B^{\prime}$ is closed in $Y, B=X \times B^{\prime}$ and $I C \Omega$, then

$$
\pi\left(B \cap \bigcap_{i \in I} A_{i}\right)=\bigcap_{i \in I} \pi\left(B \cap A_{i}\right)
$$

Proof. Apply 3.2.

(v) If $i \in \Omega$ and $i<j \in \Omega$ then

$$
\pi\left(\left(X \times \bar{U}_{i}\right) \cap A_{i}\right)=\pi\left(\left(X \times \bar{U}_{i}\right) \cap A_{j}\right)=\pi\left(\left(X \times \bar{U}_{i}\right) \cap f\right)
$$

Proof. For the first equality, we use induction on $j$. Let

$$
B=\pi\left(\left(X \times \bar{U}_{i}\right) \cap A_{i}\right)
$$

then

$$
(B \times Y) \cap A_{i+1} \subset B \times \bar{U}_{i} .
$$

Hence, in view of (ii), if $i<j \in \Omega$, we have

$$
(B \times Y) \cap A_{j} \subset\left(B \times \bar{U}_{i}\right) \cap A_{j}
$$

and

$$
\left(B \times \bar{U}_{j}\right) \cap A_{j} \subset\left(B \times \bar{U}_{i} \cap \bar{U}_{j}\right) \cap A_{j} .
$$

Now, if the first equality holds for $a$ given such $j$, then

$$
\begin{aligned}
& \pi\left(\left(X \times \bar{U}_{i}\right) \cap A_{j+1}\right) \\
&= \pi\left(\left(X \times\left(\bar{U}_{i} \cap \bar{U}_{j}\right)\right) \cap A_{j}\right) \cup\left(\pi\left(\left(X \times \bar{U}_{i}\right) \cap A_{j}\right)-\pi\left(\left(X \times \bar{U}_{j}\right) \cap A_{j}\right)\right) \\
&= \pi\left(\left(B \times\left(\bar{U}_{i} \cap \bar{U}_{j}\right)\right) \cap A_{j}\right) \cup\left(\pi\left(\left(B \times \bar{U}_{i}\right) \cap A_{j}\right)\right. \\
&\left.\quad-\pi\left(\left(B \times\left(\bar{U}_{i} \cap \bar{U}_{j}\right)\right) \cap A_{j}\right)\right) \\
&= \pi\left(\left(B \times \bar{U}_{i}\right) \cap A_{j}\right) \\
&= B
\end{aligned}
$$

so that the equality holds for $j+1$. If $j$ is a limit ordinal and the equality holds for all $j^{\prime}$ such that $i<j^{\prime}<j$, then by (iv) it also holds for $j$. The second equality follows from the first and (iv).

(vi) $\pi f=\pi Q$.

Proof. Apply (i), (iv) and induction or take $U_{0}=Y$ and apply (v). (vii) $f$ is a function. 
Proof. Suppose $(x, y) \in f$ and $\left(x, y^{\prime}\right) \in f$ and $y \neq y^{\prime}$. Let $y \in U_{i}, y^{\prime} \in U_{j}$, $\bar{U}_{i} \cap \bar{U}_{j}=0, i<j$. Then by (v) we have

$$
x \in \pi\left(\left(X \times \bar{U}_{i}\right) \cap A_{i}\right) \text { and } x \in \pi\left(\left(X \times \bar{U}_{j}\right) \cap A_{j}\right),
$$

but

$$
A_{j} \subset A_{i+1}=P\left(X \times \bar{U}_{i}, A_{i}\right) \subset\left(\left(X \times \bar{U}_{i}\right) \cap A_{i}\right) \cup((X-\{x\}) \times Y) .
$$

Hence

$$
\left(X \times \bar{U}_{j}\right) \cap A_{j} \subset(X-\{x\}) \times Y,
$$

i.e.

$$
x \notin \pi\left(\left(X \times \bar{U}_{j}\right) \cap A_{j}\right)
$$

in contradiction to the above.

(viii) If $i \in \Omega$ and $B^{\prime}$ is closed in $Y$ and $B=X \times B^{\prime}$ then $\pi\left(B \cap A_{i}\right)$ is Borel $H$.

Proof. We use induction on $i$. It is clearly true for $i=0$. Suppose it is true for $j$ and $i=j+1$. Then:

$$
\begin{aligned}
\pi\left(B \cap A_{i}\right) \\
=\pi\left(B \cap P\left(X \times \bar{U}_{j}, A_{j}\right)\right) \\
=\pi\left(B \cap\left(X \times \bar{U}_{j}\right) \cap A_{j}\right) \\
\quad \cup \pi\left(B \cap\left(\left(X-\pi\left(\left(X \times \bar{U}_{j}\right) \cap A_{j}\right)\right) \times Y\right) \cap A_{j}\right) \\
=\pi\left(B \cap\left(X \times \bar{U}_{j}\right) \cap A_{j}\right) \cup\left(\pi\left(B \cap A_{j}\right)-\pi\left(\left(X \times \bar{U}_{j}\right) \cap A_{j}\right)\right)
\end{aligned}
$$

so that $\pi\left(B \cap A_{i}\right)$ is Borel $H$.

If $i$ is a limit ordinal and the result is true for all $j<i$, by (iv), we have

$$
\pi\left(B \cap A_{i}\right)=\pi\left(\bigcap_{j<i}\left(B \cap A_{j}\right)\right)=\bigcap_{j<i} \pi\left(B \cap A_{j}\right)
$$

and the last set is Borel $H$ since the set of all $j<i$ is countable.

(ix) If $V$ is open in $Y$, then $f^{-1}(V)$ is Borel $H$.

Proof. Let

$$
I=\left\{i \in \Omega: \bar{U}_{i} \subset V\right\} .
$$

Then there exists a countable $I^{\prime} \subset I$ such that

Hence

$$
\bigcup_{i \in I^{\prime}} U_{i}=\bigcup_{i \in I} U_{i}=V .
$$

$$
\bigcup_{i \in I^{\prime}} \bar{U}_{i}=V .
$$

Then 


$$
\begin{aligned}
f^{-1}(V) & =\pi((X \times V) \cap f)=\bigcup_{i \in I^{\prime}} \pi\left(\left(X \times \bar{U}_{i}\right) \cap f\right) \\
& =(b y(\mathrm{v}))=\bigcup_{i \in I^{\prime}} \pi\left(\left(X \times \bar{U}_{i}\right) \cap A_{i}\right)
\end{aligned}
$$

so that $f^{-1}(V)$ is Borel $H$, in view of (viii).

4.2. Corollary. Suppose $X$ is any topological space; $Y$ satisfies condition (I); $Q$ is compact in $X \times Y$. Then there exists a function $f$ on $\pi Q$ such that $f \subset Q$ and, for every open set $V$ in $Y, f^{-1}(V)$ is Borel $K(X)$. (If in addition, $Q$ is metrizable then $f$ can be chosen to be of Baire first class.)

4.3. Theorem. Suppose $X$ and $Y$ are Hausdorff and $Q$ is analytic in $X \times Y$. Then there exists $Q^{\prime}$ such that: $Q^{\prime} \subset Q ; \pi Q^{\prime}=\pi Q ;$ for every $x \in X$, $\pi^{-1}\{x\} \cap Q^{\prime}$ is compact; for every closed set $B$ in $X \times Y, \pi\left(B \cap Q^{\prime}\right)$ is Borel $\mathfrak{A}(X)$.

Proof. Since $Q$ is analytic in $X \times Y$, there exists a Hausdorff space $Z$ such that $Q$ is the continuous image of a $K_{\sigma \delta}(Z)$. For every $n \in \omega$ and $i \in \omega$, let $d(n, i)$ be compact in $Z$,

$$
D=\bigcap_{n \in \omega} \bigcup_{i \in \omega} d(n, i)
$$

$f$ be a continuous function on $D$, and $Q=f(D)$. Next, let $S_{n}$ be the set of all $(n+1)$-tuples $k=\left(k_{0}, \cdots, k_{n}\right)$ with $k_{i} \in \omega$. For every $n \in \omega$ and $k \in S_{n}$, let

$$
\Delta(k)=f\left(D \cap \bigcap_{i \in(n+1)} d\left(i, k_{i}\right)\right),
$$

and then define $A(k)$ by recursion on $n$ as follows: if $k \in S_{0}$, then

$$
A(k)=\Delta(k) \cap\left(\left(X-\bigcup_{j \in k} \pi \Delta(j)\right) \times Y\right) .
$$

If $k \in S_{n+1}$, then

$$
\begin{aligned}
& A(k)=A\left(k_{0}, \cdots, k_{n}\right) \cap \Delta(k) \\
& \quad \cap\left(\left(X-\bigcup_{j \in k_{n+}} \pi\left(A\left(k_{0}, \cdots, k_{n}\right) \cap \Delta\left(k_{0}, \cdots, k_{n}, j\right)\right) \times Y\right) .\right.
\end{aligned}
$$

Finally, we set

$$
\begin{aligned}
A_{n}^{\prime} & =\bigcup_{k \in S_{n}} A(k), \\
Q^{\prime} & =\bigcap_{n \in \omega} A_{n}^{\prime},
\end{aligned}
$$

and check the following points.

(i) If $n \in \omega, k \in S_{n}, k^{\prime} \in S_{n}, k \neq k^{\prime}$, then 


$$
\pi A(k) \cap \pi A\left(k^{\prime}\right)=0 .
$$

(ii) For every $n \in \omega$ and $k \in S_{n}$,

$$
A(k) \subset \Delta(k)=\bigcup_{j \in \omega} \Delta\left(k_{0}, \cdots, k_{n}, j\right),
$$

and

$$
Q=\bigcup_{j \in \omega} \Delta(j)
$$

(iii) For every $n \in \omega, \pi A_{n}^{\prime}=\pi Q$.

Proof. We use induction on $n$. Let $x \in \pi Q$. Then by (ii)

$$
x \in \bigcup_{j \in \omega} \pi \Delta(j) \text {. }
$$

Let $j_{0}$ be the first integer such that $x \in \pi \Delta\left(j_{0}\right)$. Then,

$$
x \in \pi A\left(j_{0}\right) \subset \pi A_{0}^{\prime} .
$$

Next, suppose for some $k \in S_{n}, x \in \pi A(k)$. Then by (ii)

$$
x \in \bigcup_{j \in \omega} \pi\left(A(k) \cap \Delta\left(k_{0}, \cdots, k_{n}, j\right)\right) .
$$

Let $j_{0}$ be the first integer such that

$$
x \in \pi\left(A(k) \cap \Delta\left(k_{0}, \cdots, k_{n}, j_{0}\right)\right) .
$$

Then

$$
x \in \pi A\left(k_{0}, \cdots, k_{n}, j_{0}\right) \subset \pi A_{n+1}^{\prime} .
$$

Thus, for every $n \in \omega, \pi Q \subset \pi A_{n}^{\prime}$. The converse is immediate.

(iv) For every $x \in X$ there exists a unique sequence $k$ such that for every $n \in \omega:$

$$
\pi^{-1}\{x\} \cap A_{n}^{\prime}=\pi^{-1}\{x\} \cap A\left(k_{0}, \cdots, k_{n}\right)=\pi^{-1}\{x\} \cap \Delta\left(k_{0}, \cdots, k_{n}\right) .
$$

Proof. Use induction on $n$ and (i).

(v) If $B$ is closed in $X \times Y$ then

$$
\pi\left(B \cap Q^{\prime}\right)=\bigcap_{n \in \omega} \pi\left(B \cap A_{n}^{\prime}\right) .
$$

Proof. Suppose

$$
x \in \bigcap_{n \in \omega} \pi\left(B \cap A_{n}^{\prime}\right) .
$$

Let $k$ be the sequence given by (iv). Then for every $n \in \omega$ :

$$
0 \neq \pi^{-1}\{x\} \cap B \cap A_{n}^{\prime}=\pi^{-1}\{x\} \cap B \cap \Delta\left(k_{0}, \cdots, k_{n}\right) .
$$


Since $f$ is continuous on $D$ and $\pi^{-1}\{x\} \cap B$ is closed, there exists a closed set $C$ in $Z$ such that

$$
f^{-1}\left(\pi^{-1}\{x\} \cap B\right)=D \cap C .
$$

Recalling the definition of $\Delta\left(k_{0}, \cdots, k_{n}\right)$ we conclude

$$
D \cap C \cap \bigcap_{i \in(n+1)} d\left(i, k_{i}\right) \neq 0 .
$$

Since the $d\left(i, k_{i}\right)$ are compact, we have

$$
\bigcap_{n \in \omega}\left(C \cap \bigcap_{i \in(n+1)} d\left(i, k_{i}\right)\right) \neq 0
$$

Therefore

$$
\pi^{-1}\{x\} \cap B \cap f\left(\bigcap_{n \in \omega} \bigcap_{i \in(n+1)} d\left(i, k_{i}\right)\right) \neq 0
$$

and, by 3.1 ,

$$
\pi^{-1}\{x\} \cap B \cap \bigcap_{n \in \omega} f\left(D \cap \bigcap_{i \in(n+1)} d\left(i, k_{i}\right)\right) \neq 0
$$

i.e.,

$$
\bigcap_{n \in \omega}\left(\pi^{-1}\{x\} \cap B \cap \Delta\left(k_{0}, \cdots, k_{n}\right)\right) \neq 0
$$

Hence, by (iv)

$$
\bigcap_{n \in \omega}\left(\pi^{-1}\{x\} \cap B \cap A_{n}^{\prime}\right) \neq 0
$$

i.e.,

$$
x \in \pi \bigcap_{n \in \omega}\left(B \cap A_{n}^{\prime}\right)=\pi\left(B \cap Q^{\prime}\right) .
$$

Thus,

$$
\bigcap_{n \in \omega} \pi\left(B \cap A_{n}^{\prime}\right) \subset \pi\left(B \cap Q^{\prime}\right) .
$$

The converse is immediate.

(vi) If $B$ is either closed or analytic in $X \times Y$ then, for every $n \in \omega$ and $k \in S_{n}, \pi(B \cap A(k))$ is Borel $\mathfrak{Q}(X)$.

Proof. We use induction on $n$. We first observe that the intersection of two analytic sets, the intersection of an analytic set with a closed set, and the continuous image of an analytic set are all analytic. Thus, for every $n \in \omega$ and $k \in S_{n}, \pi(B \cap \Delta(k))$ is analytic in $X$. Now, if $k \in S_{0}$, since

$$
\pi(B \cap A(k))=\pi(B \cap \Delta(k))-\bigcup_{j \in k} \pi \Delta(j)
$$


we see that $\pi(B \cap A(k))$ is the difference of two analytic sets in $X$. Next, suppose the result holds for a given $n \in \omega$ and let $k \in S_{n+1}$. Then

$$
\pi\left(B \cap \Delta\left(k_{0}, \cdots, k_{n}, j\right) \cap A\left(k_{0}, \cdots, k_{n}\right)\right)
$$

is Borel $\mathfrak{A}(X)$ for every $j \in \omega$, and since

$$
\begin{gathered}
\pi(B \cap A(k))=\pi\left(B \cap \Delta(k) \cap A\left(k_{0}, \cdots, k_{n}\right)\right) \\
\quad-\bigcap_{j \in k_{n+1}} \pi\left(A\left(k_{0}, \cdots, k_{n}\right) \cap \Delta\left(k_{0}, \cdots, k_{n}, j\right)\right),
\end{gathered}
$$

we see that $\pi(B \cap A(k))$ is the difference of two Borel $\mathfrak{A}(X)$ sets.

(vii) For every closed set $B$ in $X \times Y, \pi\left(B \cap Q^{\prime}\right)$ is Borel $\mathfrak{A}(X)$.

Proof. By (v) we have:

$$
\pi\left(B \cap Q^{\prime}\right)=\bigcap_{n \in \omega} \pi\left(B \cap A_{n}^{\prime}\right)=\bigcap_{n \in \omega} \bigcup_{k \in S_{n}} \pi(B \cap A(k)) .
$$

Since $S_{n}$ is countable, the result follows from (vi).

(viii) For every $x \in X, \pi^{-1}\{x\} \cap Q^{\prime}$ is compact.

Proof. Let $k$ be the sequence given by (iv). Then

$$
\begin{aligned}
\pi^{-1}\{x\} \cap Q^{\prime} & =\bigcap_{n \in \omega}\left(\pi^{-1}\{x\} \cap A_{n}^{\prime}\right)=\bigcap_{n \in \omega}\left(\pi^{-1}\{x\} \cap \Delta\left(k_{0}, \cdots, k_{n}\right)\right) \\
& =\pi^{-1}\{x\} \cap \bigcap_{n \in \omega} f\left(D \cap \bigcap_{i \in(n+1)} d\left(i, k_{i}\right)\right)=(\text { by 3.1) } \\
& =\pi^{-1}\{x\} \cap f\left(\bigcap_{i \in \omega} d\left(i, k_{i}\right)\right)
\end{aligned}
$$

so that $\pi^{-1}\{x\} \cap Q^{\prime}$ is the intersection of a closed set with a compact set.

4.4. Corollary. Suppose $X$ is Hausdorff, $Y$ satisfies condition (I), and $Q$ is analytic in $X \times Y$. Then there exists a function $f$ on $\pi Q$ such that $f \subset Q$ and, for every open set $V$ in $Y, f^{-1}(V)$ is Borel $\mathfrak{A}(X)$.

4.5. Corollary. Suppose $X$ is Hausdorff, $Y$ satisfies condition (I), and $Q$ is analytic in $X \times Y$. Then there exists a function $f$ on $\pi Q$ such that $f \subset Q$ and, for every open set $V$ in $Y, f^{-1}(V)$ is $\mu$-measurable for all Caratheodory outer measures $\mu$ on $X$ such that closed sets are $\mu$-measurable.

Proof. In this case (see [7]), the analytic sets in $X$ can be obtained by applying Souslin's operation $a$ to the family of closed sets in $X$. Hence they are $\mu$-measurable whenever closed sets are $\mu$-measurable. (See e.g., S. Saks, Theory of the integral, p. 50.)

\section{BIBLIOGRAPHY}

1. N. Bourbaki, Eléments de mathêmatique, VIII, Part I, Vol. III, Topologie générale, Chapter 9, Paris, 1958, p. 135. 
2. M. Kondô, Sur l'uniformisation des complémentaires analytiques et les ensembles projectifs de la seconde classe, Jap. J. Math. vol. 15 (1938) pp. 197-230.

3. N. Luzin, Sur le problème de M. J. Hadamard d'uniformisation des ensembles, Mathematica vol. 4 (1930) pp. 54-66.

4. E. Michael, Continuous selections I, Ann. of Math. (2) vol. 63 (1956) pp. 361-382.

5. W. Sierpinski, Les ensembles projectifs et analytiques, Mémor. Sci. Math., vol. 12, 1950.

6. - Sur l'uniformisation des ensembles mesurables (B), Fund. Math. vol. 16 (1930) pp. $136-139$.

7. M. Sion, On analytic sets in topological spaces, Trans. Amer. Math. Soc. vol. 96 (1960) pp. 341-354.

8. M. H. Stone and J. von Neumann, The determination of representative elements in the residual classes of a Boolean algebra, Fund. Math. vol. 25 (1935) pp. 353-378.

UNIVERSity OF California,

Berkeley, California 